\title{
The systemic-immune-inflammation index predicts the recurrence of atrial fibrillation after cryomaze concomitant with mitral valve surgery
}

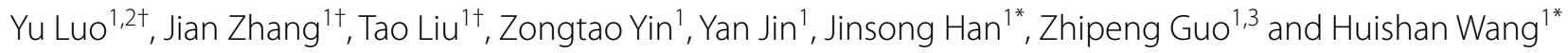

\begin{abstract}
Background and aims: Inflammation plays a key role in the initiation and progression of atrial fibrillation (AF). The systemic inflammation indexes are easily evaluated and predict AF development. However, it's role in prediction of recurrence of $A F$ is unknown. We aim to explore the association between the systemic inflammation indexes and recurrence of AF in patients underwent cryoablation (CryoMaze) concomitant with mitral valve surgery.
\end{abstract}

Methods: We examined systemic inflammation indexes during perioperative period in 122 patients between 2015 and 2018. Systemic inflammation indexes were developed by systemic immune-inflammation index (SII), neutrophil to lymphocyte ratio (NLR), platelet to lymphocyte ratio (PLR), and lymphocytes to monocytes ratio. Univariate and multivariate analyses were performed to examine the association of each markers with recurrence of AF.

Results: Of the 122 patients included in this study, 22 patients (18\%) experienced AF recurrence after CryoMaze concomitant with mitral valve surgery. There is no significant difference between each systemic inflammation indexes before surgery and recurrence of AF. In univariate analysis, MLR after surgery 3 days, PLR, MPLR, NLR, SII after surgery 7 days were able to predict recurrence of AF. In multivariate analyses, SII $\geq 1696$ independently predicted recurrence (OR, 3.719; 95\% Cl, 1.417-9.760). Interestingly, baseline SIl showed no significant in prediction of recurrence. It was sharply elevated after surgery and dropped slowly. In patients of recurrence, SII after 7 days of surgery increased again.

Conclusions: The raised SII again was associated with an increased risk of the postoperative recurrence of AF and independently predicted the late recurrence of AF after CryoMaze concomitant with mitral valve surgery.

Keywords: Systemic-immune-inflammation index, Recurrence of atrial fibrillation, CryoMaze, Systemic immuneinflammation index

*Correspondence: hanjs0216@sina.com; huishanw@126.com

${ }^{\dagger}$ Yu Luo, Jian Zhang and Tao Liu contributed equally to this work

1 Department of Cardiovascular Surgery, General Hospital of Northern

Theater Command, No.83, Wenhua Road, Shenhe District,

Shenyang 110016, Liaoning, China

Full list of author information is available at the end of the article

\section{Introduction}

Atrial fibrillation (AF) is a common arrhythmia associated with mitral valve disease. Nearly half of patients undergoing mitral valve surgery frequently present with AF, which is associated with poor prognosis [1]. The Cox-Maze IV operation is widely used in the surgical treatment of AF [2]. However, the efficiency was various [3-5]. Our recent study indicated that the efficiency of 
cryoablation (CryoMaze) concomitant with mitral valve surgery was achieved in $85 \%$ (95\% CI, 0.76-0.91) [5].

The pathophysiological mechanisms of AF are not well known [6]. Inflammation and substrate alterations (such as fibrosis) are complex and critical for the understanding of AF [7-10]. The mechanism of recurrences may because of incomplete isolation, acute inflammatory changes, recovery of conduction and modification of autonomic nervous system [11]. The systemic inflammation index is a systemic inflammation based on routine blood tests [12]. Neutrophil to lymphocyte ratio (NLR), platelet to lymphocyte ratio and lymphocyte to monocyte ratio (LMR) are reported to be associated with systemic inflammation status and AF progression [13]. Systemic immune-inflammation index (SII) is a novel marker that brings together these three inflammatory peripheral cell counts and predict functionally significant coronary artery stenosis [14-16]. In this study, we investigated the association of perioperative systemic inflammation index, and changes during treatment, with outcomes in AF patients treated with CryoMaze concomitant with mitral valve surgery.

\section{Methods}

\section{Patients' selection}

We retrospectively studied consecutive 150 longpersistent or persistent $\mathrm{AF}$ patients who required to undergoing the cryoMaze procedures combined with valve-surgery at the department of cardiovascular surgery, General Hospital of Northern Theatre Command, from 2015 to 2018. Exclusion criteria were (1) emergency surgery; (2) combined with CABG or any other heart procedures; (3) previous cardiovascular surgery; (4) primary pulmonary hypertension; (5) $\mathrm{EF}<0.40$, and (6) history of cerebral hemorrhage or brain stoke within 3 months. Of these, 122 (81.3\%) had baseline complete blood counts necessary for the inflammatory indexes and all clinical data available and were considered fully evaluable for this post hoc analysis.

This study was approved by the General Hospital of Northern Theatre Command's Ethics committee. All procedures were performed by the Declaration of Helsinki and its later amendments or comparable ethical standards.

\section{Surgical procedure, ablation procedure, post-operative management, and follow-up}

Surgical procedure, ablation procedure, post-operative management and follow-up have been described before [5]. Briefly, after cardioplegic arrest and aortic crossclamping, the left lesion sets and concomitant operations were performed. The lesion sets of the CryoMaze were created using cryothermia based on Nitrous Oxide
(Atricure CRYO2 Cryosurgical probe, $-60{ }^{\circ} \mathrm{C}, 2 \mathrm{~min}$ ). The detail of lesion sets, and ablation procedure could be found before [5].

Heart rhythm was monitored continuously throughout the hospital stay, and temporary epicardial wires were used for ventricular pacing as needed in all the patients after the surgery. Amiodarone was given intravenously from 20 to $40 \mathrm{mg} / \mathrm{h}$, followed by oral amiodarone at $200 \mathrm{mg}$ twice a day and then $200 \mathrm{mg} /$ day until 3 months after discharge. According to the 24-h Holter results at 3 months, amiodarone was withdrawn for patients restore to sinus rhythm. Amiodarone was continually administered in AF patients. Electrical cardioversion was applied in patients when oral amiodarone failed to maintain sinus rhythm. After discharge, patients were followed up at outpatient clinic at 1, 3, 6, and 12 months. Heart rhythm was verified with 24-h Holter monitoring and echocardiography were evaluated by 2 -dimensional echocardiographic analysis and Doppler color flow imaging (Philips iE33 ultrasound machine; Philips Healthcare, Andover, Mass) at each visit [5]. The definition of atrial tachyarrhythmia recurrence was any documented AF, atrial flutter, or atrial tachyarrhythmia lasting $\geq 30 \mathrm{~s}$ after 3 months blanking period [17].

\section{Post hoc analysis variable definitions}

For this post hoc analysis, the inflammatory indexes were determined based on values of monocytes $(\mathrm{M})$, neutrophils $(\mathrm{N})$, lymphocytes $(\mathrm{L})$, and/or platelets $(\mathrm{P})$ in patients received surgical ablation at indicated data: SII defined as $\mathrm{P} \times \mathrm{N} / \mathrm{L}$, NLR defined as N/L, platelet-lymphocyte ratio (PLR) defined as $\mathrm{P} / \mathrm{L}$, lymphocyte to monocyte ratio (LMR) defined as L/M, monocytes-NLR (MNLR) defined as $\mathrm{M} \times \mathrm{N} / \mathrm{L}$, monocytes-PLR (MPLR) defined as $\mathrm{M} \times \mathrm{P} / \mathrm{L}$ $[14,18,19]$.

\section{Statistical analysis}

The IBM SPSS.24.0 software was used for all dates analyzed. Continuous variables were presented as Mean \pm SD or medians (range). And the categorical variables were described as frequencies and percentages. To compare the differences between two groups, the independent student's $t$ text and Mann-Whitney U text were used. Chi-squared test or Fisher's exact test were used for categorical variables. Risk factors that $p<0.05$ were included in the Univariate logistic regression analysis. Receiver operating characteristic curves (ROC) were generated to provide data on the predictive ability of the systemic inflammation indexes to detect recurrence of AF. The area under the curve (AUC) was used to quantify the ROC curve. Youden's index $(J=$ Sensitivity + Specificity -1 ) was used to determine the most appropriate 
Table 1 Baseline and clinical characteristics $(N=122)$

\begin{tabular}{|c|c|c|c|}
\hline & \multicolumn{2}{|c|}{ Rhythm after surgery (12 m) } & \multirow[t]{2}{*}{$P$} \\
\hline & Recurrence versus & Non-recurrence & \\
\hline Age & $59.640 \pm 6.403$ & $59.690 \pm 8.781$ & 0.978 \\
\hline $\begin{array}{l}\text { Gender (\%) } \\
\text { (Male versus female) }\end{array}$ & 11 (50.0) versus $9(50.0)$ & $32(32.0)$ versus 68 (68.0) & 0.110 \\
\hline Degenerative disease (\%) & $9(40.9)$ & $47(47.0)$ & 0.604 \\
\hline Hypertension (\%) & $4(18.2)$ & $13(13.0)$ & 0.768 \\
\hline Coronary arteries disease (\%) & $6(27.3)$ & $28(28.0)$ & 0.945 \\
\hline Diabetes (\%) & $2(9.1)$ & $5(5.0)$ & 0.810 \\
\hline Kidneys disease (\%) & $0(0)$ & $3(3.0)$ & 1.000 \\
\hline NYHA III (\%) & $15(68.2 \%)$ & $70(70.0 \%)$ & 0.867 \\
\hline $\mathrm{LAD}(\mathrm{mm})$ & $54.500 \pm 9.787$ & $51.600 \pm 8.661$ & 0.168 \\
\hline LAVI $\left(\mathrm{ml} / \mathrm{m}^{2)}\right.$ & $47.320 \pm 8.231$ & $45.080 \pm 7.782$ & 0.229 \\
\hline $\operatorname{LVEDD}(\mathrm{mm})$ & $50.590 \pm 6.345$ & $47.750 \pm 6.363$ & 0.060 \\
\hline LVEDV (ml) & $153.140 \pm 147.128$ & $111.000 \pm 36.166$ & 0.196 \\
\hline LVEF (\%) & $54.000 \pm 5.800$ & $56.000 \pm 4.200$ & 0.192 \\
\hline $\mathrm{ACC}(\mathrm{min})$ & $83.180 \pm 25.299$ & $87.440 \pm 31.255$ & 0.552 \\
\hline CPB (min) & $143.820 \pm 35.391$ & $146.540 \pm 42.790$ & 0.782 \\
\hline
\end{tabular}

LAD left atrium diameter, LAVI left atrial volume indexed, LVEDD left ventricular end diastolic diameter, LVEDV left ventricular end diastolic volume, LVEF left ventricular ejection fraction, $A C C$ aortic clip, $C P B$ cardiopulmonary bypass

cut-off value. $p<0.05$ was considered significant in all comparisons.

\section{Results}

\section{Study population}

A total of 122 patients who underwent CryoMaze concomitant with mitral valve surgery were included in this study. Recurrence occurred in 22 patients (18.0\%). Table 1 summarizes the characteristics of the cohort based on recurrence or not. Preoperative left atrial diameter (LAD) $(p=0.196)$ and left atrial volume indexed (LAVI) $(p=0.229)$ are elevated in patients have AF recurrence, but there was no statistical significance. All of patients were diagnosed with NYHA III $(\mathrm{N}=100)$ or IV $(\mathrm{N}=22)$ and there was no significance between two groups.

\section{Systemic inflammation index and recurrence}

The systemic inflammation index before surgery was no related with recurrence (Additional file 1: Table S1). Furthermore, the similar results were found in the early after the surgery (Additional file 1: Tables S2, S3 and S4). Interestingly, NLR, PLR, MPLR and SII levels after 7 days of surgery were significantly higher in recurrence group $(p=0.033 ; p=0.050 ; p=0.042 ; p=0.024)$ (Table 2$)$. Furthermore, we found that in recurrence patients SII dropped along with no-recurrence patients after 1 day of surgery but elevated at 7 days after the surgery (Fig. 1).
Table 2 Inflammatory markers of day 7 in post-operative

\begin{tabular}{lccl}
\hline & \multicolumn{2}{l}{ Rhythm after surgery $(\mathbf{1 2} \mathbf{~ m})$} & $\boldsymbol{P}$ \\
\cline { 2 - 3 } & Recurrence versus & Non-recurrence & \\
\hline NLR-7 & $6.869 \pm 2.615$ & $5.667 \pm 2.310$ & $\mathbf{0 . 0 3 3}$ \\
MLR-7 & $0.619 \pm 0.257$ & $0.568 \pm 0.197$ & 0.301 \\
PLR-7 & $241.901 \pm 99.112$ & $201.624 \pm 83.667$ & $\mathbf{0 . 0 5 0}$ \\
MPLR-7 & $185.928 \pm 100.588$ & $150.150 \pm 66.969$ & $\mathbf{0 . 0 4 2}$ \\
MNLR-7 & $5.456 \pm 2.962$ & $4.452 \pm 2.402$ & 0.092 \\
SII-7 & $2137.871 \pm 1150.615$ & $1520.137 \pm 786.779$ & $\mathbf{0 . 0 2 4}$ \\
ALB-7 & $33.031 \pm 2.352$ & $33.960 \pm 3.647$ & 0.256 \\
\hline
\end{tabular}

NLR-7 the NLR of post-day $7, M L R-7$ the MLR of post-day 7, PLR-7 the PLR of postday 7, MPLR-7 the MPLR of post-day 7, MNLR-7 the MNLR of post-day 7, SII-7 the SIl of post-day $7, A L B-7$ the albumin of post-day 7

Bold values indicated $p<0.05$

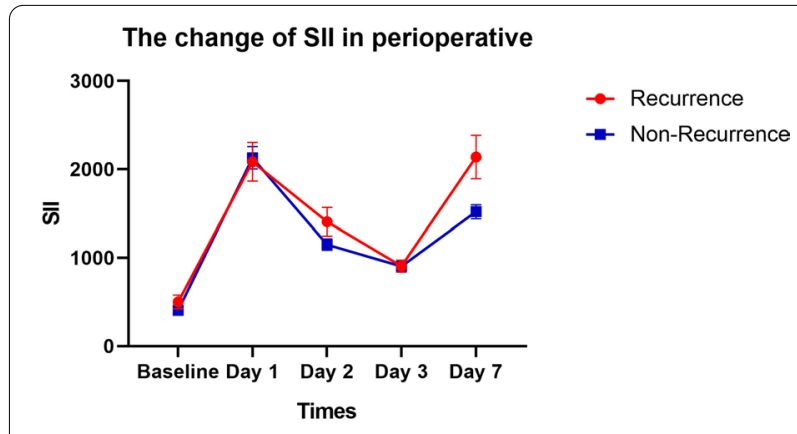

Fig. 1 The tendency of SII before and after surgery at indicated days 
We used SII at 7 days after surgery data for receiver operating characteristic (ROC) analysis. The area under the ROC curve was 0.680 , and the $95 \%$ confidence interval was $0.566-0.808$ (Fig. 2). A cut-off points of 1696 was related to recurrence with a sensitivity of $63.6 \%$ and a specificity of $68.0 \%$.

The distribution of characteristics in cohort and SII levels after 7 days of surgery is listed in Additional file 1: Table S5. However, there was no correlation between high SII levels after 7 days of surgery and other variables (Additional file 1: Table S5).

\section{Univariate and multivariate analysis of recurrence}

On multivariable logistic regression analysis, high SII after 7 days of surgery (OR, 3.719 (1.417-9.760), $p=0.008)$ were significant and independent risk factors for recurrence (Table 3).

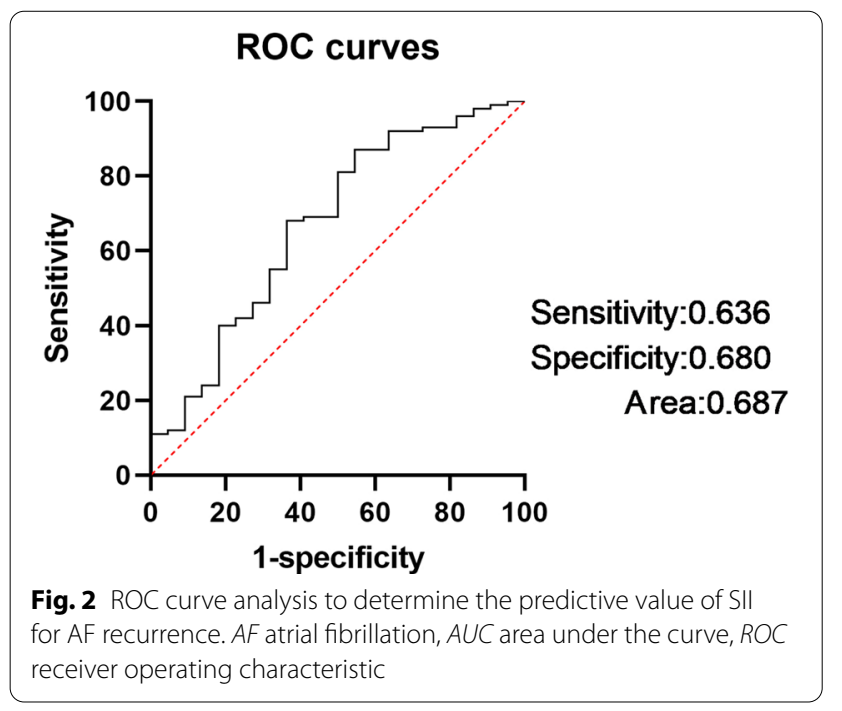

\section{Discussion}

In our retrospective study, we focused more on the relationship between the systemic inflammation index and development of AF recurrence after CryoMaze concomitant with mitral valve surgery. We found that SII was increased immediately after the surgery and dropped within several days. Interestingly, in recurrence patients, SII levels elevated at 7 days after surgery again. Additionally, we had demonstrated that the SII are independent prognostic factor of AF recurrence in patients who had undergone CryoMaze concomitant with mitral valve surgery.

The precise mechanisms of AF recurrence have not been fully elucidated. it is well established that inflammation is independently associated with the development and recurrent of AF [20]. Several studies indicated markers of inflammation predicted recurrence of $\mathrm{AF}[21,22]$. The atrial tissue changes such as fibrosis, leukocyte infiltrates and oxidative damage may contribute to electrical and structural remodeling, which may promote recurrence of AF [23]. Some inflammatory biomarkers, including CRP, IL-6, IL-2, matrix metalloproteinase-2 and tumor necrosis factor- $\alpha$, were found to be significantly associated with recurrent $\operatorname{AF}[21,24,25]$. Moreover, antiinflammatory therapy may reduce the recurrence $\mathrm{AF}$ [26].

The SII can better reflect the immune and inflammatory state of the body compared to the use of any one of these markers in isolation [27]. It has been widely studied in different cancers [12, 27]. What is more, levels of neutrophils and lymphocytes are related with initiation and progression of AF [28-30]. Inflammatory mediators, released by neutrophils, such as interleukin-8, matrix metalloproteinase-9, and vascular endothelial growth factor promoted AF. Recently, platelets was reported the profibrotic actions through Transforming Growth Factor- $\beta 1$ dependent manners [31]. SII not only contained the ratio of neutrophil and lymphocyte ratio, but

Table 3 Univariable and multivariable logistic regression of SII-7

\begin{tabular}{|c|c|c|c|c|c|c|}
\hline & \multicolumn{3}{|c|}{ Univariable } & \multicolumn{3}{|c|}{ Multivariable } \\
\hline & OR & $p$ & $\mathrm{Cl}$ & OR & $p$ & $\mathrm{Cl}$ \\
\hline High MLR-3 & 2.855 & 0.032 & $1.096-7.442$ & & & \\
\hline High PLR-7 & 3.127 & 0.053 & $0.986-9.919$ & & & \\
\hline HighMPLR-7 & 3.380 & 0.017 & $1.247-9.163$ & & & \\
\hline High NLR-7 & 3.810 & 0.008 & $1.422-10.208$ & & & \\
\hline High SII-7 & 3.719 & 0.008 & $1.417-9.760$ & 3.719 & 0.008 & $1.417-9.760$ \\
\hline
\end{tabular}

High MLR-3 the MLR of postoperative day $3 \geq 0.63961$, High PLR-7 the PLR of postoperative day $7 \geq 174.2083$, High MPLR-7 the MPLR of postoperative day $7 \geq 189.9917$, High NLR-7 the NLR of postoperative day $7 \geq 5.913194$, High SII-7 the SII of postoperative day $7 \geq 1696.005051$, OR odds ratio, Cl confidence interval; The variables of Multivariable are High MLR-3, HighMPLR-7, High NLR-7, High SII-7

Bold values indicated $p<0.05$ 
contained the levels of platelets, which may reflect atrial fibrosis to some extent. Therefore, SII is superior to other systemic inflammation index in prognostic assessment of AF ablation in our cohort.

$\mathrm{AF}$ is the most common cardiac arrhythmia worldwide. In patients underwent valve surgery, the prevalence of AF is approximately $30-50 \%$ [5]. The cut-and-sew Maze procedure is considered the gold standard for the surgical treatment of AF [32]. Because of its complexity and perceived risks of bleeding, various energy sources such as radiofrequency and cryoablation to simplify surgical AF ablation. Previously, our group reported the addition of the Maze performed by cryoablation (CryoMaze) was non-inferior to CSM for efficacy and safety for patients with persistent or long-standing persistent AF undergoing mitral valve surgeries [5]. The efficacy reported by us is similar with that reported by other groups [33]. So, the recurrence of AF is not blame for our procedure. Many evidences indicated acute inflammatory changes is essential for recurrence of AF [11]. Our results clearly showed SII was elevated at 1 day after the surgery, then it dropped significantly. However, in patients who would fail to ablation, SII was elevated again. Our finding supported inflammatory changes promoted AF recurrence. On the other hand, targeting local inflammation is a therapeutic option for preventing recurrences of AF. Although, inflammation is not the only factor responsible for triggering recurrences of AF [34]. Anti-inflammatory such as colchicine were consistent and promising for $\mathrm{AF}$ recurrence [34]. All this evidence indicated targeting inflammatory could be one method for AF recurrence in patients treated with CryoMaze concomitant with mitral valve surgery.

This was a single center retrospective analysis of a small patient cohort. In addition, the relatively small sample size in this study limited the statistical capacity. Further prospective studies would be need to confirmation of these findings with large sample size.

In conclusion, SII as an inflammatory marker is associated with an independent increased risk of AF recurrence among patients underwent CryoMaze concomitant with mitral valve surgery. Future research is needed to elucidate the specific mechanism of inflammatory biomarkers in the recurrence of AF.

\footnotetext{
Abbreviations

AF: Atrial fibrillation; CryoMaze: Cryoablation; LAD: Left atrial diameter; LMR: Lymphocytes to monocytes ratio; LVEDD: Left ventricular end diastolic diameter; NLR: Neutrophil to lymphocyte ratio; PLR: Platelet to lymphocyte ratio; ROC: Receiver operating characteristic; SII: Systemic immune-inflammation index.
}

\section{Supplementary Information}

The online version contains supplementary material available at https://doi. org/10.1186/s12872-022-02494-z.

Additional file 1: Inflammatory markers in per-operative period.

Acknowledgements

Not applicable.

Authors' contributions

$Y L, J Z$ and $T L$ wrote the main manuscript text and $Y L, Y J$ and $Z Y$ prepared Figs. 1 and 2, ZG, JH and YL prepared Tables 1, 2 and 3. All authors reviewed the manuscript. All authors read and approved the final manuscript.

\section{Funding}

This work was supported by grants from the National Natural Sciences Fund Project of China $(81771833,81970310,82070239$ and 82170328), Provincial Key R \& D Program (2019JH2/10300002, 2021JH2/10300082 and 2019JH8/10300084), National Scientific Research Foundation of Liaoning Province in China (20180550959 and 2020-MS-036) and Young Talent Fund of Military Science and Technology Project (18-JCJQ-QT-018).

\section{Availability of data and materials}

The essential data are available from the corresponding author on reasonable request.

\section{Declarations}

\section{Ethics approval and consent to participate}

The study was approved by our institutional review board (the Ethics Committee of the General Hospital of Northern Theater Command.), which waived the requirement for individual patient consent because only routine patient data were used for this retrospective analysis. All experiments were performed in accordance with relevant guidelines and regulations.

\section{Consent for publication}

Not applicable.

\section{Competing interests}

The authors declare that they have no competing interests.

\section{Author details}

${ }^{1}$ Department of Cardiovascular Surgery, General Hospital of Northern Theater Command, No.83, Wenhua Road, Shenhe District, Shenyang 110016, Liaoning, China. ${ }^{2} \mathrm{CCU}$ of Surgery, Wuhan Asia Heart Hospital, Jianghan District, Wuhan 430040, Hubei, China. ${ }^{3}$ Postgraduate Training Base of Northern Theater Command General Hospital, China Medical University, No.83, Wenhua Road, Shenhe District, Shenyang 110016, Liaoning, China.

Received: 10 February 2021 Accepted: 7 February 2022

Published online: 13 February 2022

\section{References}

1. Wang H, Han J, Wang Z, Yin Z, Liu Z, Jin Y, Han H. Efficacy of cut-andsew surgical ablation for atrial fibrillation in patients with giant left atria undergoing mitral valve surgery: a propensity-matched analysis. Semin Thorac Cardiovasc Surg. 2019;31(4):796-802.

2. Ad N. The Cox-Maze procedure: history, results, and predictors for failure. J Interv Cardiac Electrophysiol Int J Arrhythm Pacing. 2007;20(3):65-71.

3. Gillinov AM, Gelijns AC, Parides MK, DeRose JJ Jr, Moskowitz AJ, Voisine P, Ailawadi G, Bouchard D, Smith PK, Mack MJ, et al. Surgical 
ablation of atrial fibrillation during mitral-valve surgery. N Engl J Med. 2015;372(15):1399-409.

4. Ad N, Suri RM, Gammie JS, Sheng S, O'Brien SM, Henry L. Surgical ablation of atrial fibrillation trends and outcomes in North America. J Thorac Cardiovasc Surg. 2012;144(5):1051-60.

5. Han J, Wang H, Wang Z, Yin Z, Zhang J, Jin Y, Han H: Comparison of cryomaze with cut-and-sew maze concomitant with mitral valve surgery: a randomized noninferiority trial. LID - S1043-0679(20)30423-8 [pii] LID. https://doi.org/10.1053/j.semtcvs.2020.11.028. 2020 (1532-9488 (Electronic)).

6. Sagnard A, Hammache N, Sellal JM, Guenancia C. New perspective in atrial fibrillation. J Clin Med. 2020;9(11):3713.

7. Guenancia C, Toucas C, Fauchier L, Stamboul K, Garnier F, Mouhat B, Sagnard A, Lorgis L, Zeller M, Cottin Y. High rate of recurrence at longterm follow-up after new-onset atrial fibrillation during acute myocardial infarction. (1532-2092 (Electronic)).

8. Zhang J, Xu S, Xu Y, Liu Y, Li Z, Zhang Y, Jin Y, Xue X, Wang H. Relation of mitochondrial DNA copy number in peripheral blood to postoperative atrial fibrillation after isolated off-pump coronary artery bypass grafting. (1879-1913 (Electronic)).

9. Li Z, Wang Z, Yin Z, Zhang Y, Xue X, Han J, Zhu Y, Zhang J, Emmert MY, Wang H. Gender differences in fibrosis remodeling in patients with longstanding persistent atrial fibrillation. Oncotarget. 2017;8(32):53714-29.

10. Xue $X D$, Huang $J H$, Wang $H S$. Angiotensin II activates signal transducers and activators of transcription 3 via Rac1 in the atrial tissue in permanent atrial fibrillation patients with rheumatic heart disease. Cell Biochem Biophys. 2015;71(1):205-13.

11. Stabile G, lacopino S, Verlato R, Arena G, Pieragnoli P, Molon G, Manfrin M, Rovaris G, Curnis A, Bertaglia E, et al. Predictive role of early recurrence of atrial fibrillation after cryoballoon ablation. Europace Eur Pacing Arrhythm Cardiac Electrophysiol J Working Groups Cardiac Pacing Arrhythm Cardiac Cell Electrophysiol Eur Soc Cardiol. 2020;22(12):1798-804.

12. De Giorgi U, Procopio G, Giannarelli D, Sabbatini R, Bearz A, Buti S, Basso U, Mitterer M, Ortega C, Bidoli P, et al. Association of systemic inflammation index and body mass index with survival in patients with renal cell cancer treated with nivolumab. Clin Cancer Res Off J Am Assoc Cancer Res. 2019;25(13):3839-46.

13. Zhang H, Li J, Chen X, Wu N, Xie W, Tang H, Li C, Wu L, Xiang Y, Zhong L, et al. Association of systemic inflammation score with atrial fibrillation: a case-control study with propensity score matching. Heart Lung Circ. 2018;27(4):489-96.

14. Erdogan M, Erdol MA, Ozturk S, DurmazT. Systemic immune-inflammation index is a novel marker to predict functionally significant coronary artery stenosis. Biomark Med. 2020;14(16):1553-61.

15. Kelesoglu S, Yilmaz Y, Elcik D, Kalay N. Systemic immune inflammation index: a novel predictor for coronary collateral circulation. Perfusion 2021:2676591211014822.

16. Kelesoglu S, Yilmaz Y, Elcik D, Cetinkaya Z, Inanc MT, Dogan A, Oguzhan A, Kalay N. Systemic immune inflammation index: a novel predictor of contrast-induced nephropathy in patients with non-ST segment elevation myocardial infarction. Angiology. 2021;72(9):889-95.

17. Wu Z, Jiang CA-O, Li J, Du J, Bai Y, Guo X, Wang W, Li S, Jiang C, Liu NA-O et al. Effect of family history of atrial fibrillation on recurrence after atrial fibrillation ablation: a report from the Chinese atrial fibrillation registry study. LID—https://doi.org/10.1111/jce.14919 [doi]. (1540-8167 (Electronic)).

18. Zhang H, Li J, Chen X, Wu N, Xie W, Tang H, Li C, Wu L, Xiang Y, Zhong $L$ et al. Association of systemic inflammation score with atrial fibrillation: a case-control study with propensity score matching. (1444-2892 (Electronic)).

19. Liu X, Ge H, Feng X, Hang J, Zhang F, Jin X, Bao H, Zhou M, Han F, Li S, et al. The combination of hemogram indexes to predict exacerbation in stable chronic obstructive pulmonary disease. Front Med. 2020;7:572435.

20. Calkins H, Hindricks G, Cappato R, Kim YH, Saad EB, Aguinaga L, Akar JG, Badhwar V, Brugada J, Camm J, et al. 2017 HRS/EHRA/ECAS/APHRS/ SOLAECE expert consensus statement on catheter and surgical ablation of atrial fibrillation. Heart Rhythm. 2017;14(10):e275-444.

21. Meyre PA-O, Sticherling C, Spies F, Aeschbacher S, Blum S, Voellmin G, Madaffari A, Conen D, Osswald S, Kühne M et al. C-reactive protein for prediction of atrial fibrillation recurrence after catheter ablation. (1471-2261 (Electronic)).
22. Chen SA, Zhang MM, Zheng M, Liu F, Sun L, Bao ZY, Chen FK, Li HX, Gu $X A-O$. The preablation monocyte/ high density lipoprotein ratio predicts the late recurrence of paroxysmal atrial fibrillation after radiofrequency ablation. (1471-2261 (Electronic)).

23. McGarry TJ, Narayan SM. The anatomical basis of pulmonary vein reconnection after ablation for atrial fibrillation: wounds that never felt a scar? J Am Coll Cardiol. 2012:59(10):939-41.

24. Cabrera-Bueno F, Medina-Palomo C, Ruiz-Salas A, Flores A, RodriguezLosada N, Barrera A, Jimenez-Navarro M, Alzueta J. Serum levels of interleukin-2 predict the recurrence of atrial fibrillation after pulmonary vein ablation. Cytokine. 2015;73(1):74-8.

25. Kimura T, Takatsuki S, Inagawa K, Katsumata Y, Nishiyama T, Nishiyama N, Fukumoto $K$, Aizawa $Y$, Tanimoto $Y$, Tanimoto $K$, et al. Serum inflammation markers predicting successful initial catheter ablation for atrial fibrillation. Heart Lung Circ. 2014;23(7):636-43.

26. Koyama T, Tada H, Sekiguchi Y, Arimoto T, Yamasaki H, Kuroki K, Machino T, Tajiri K, Zhu XD, Kanemoto-Igarashi M, Sugiyasu A: Prevention of atrial fibrillation recurrence with corticosteroids after radiofrequency catheter ablation: a randomized controlled trial. (1558-3597 (Electronic)).

27. Aziz MH, Sideras K, Aziz NA, Mauff K, Haen R, Roos D, Saida L, Suker M, van der Harst E, Mieog JS et al. The systemic-immune-inflammation index independently predicts survival and recurrence in resectable pancreatic cancer and its prognostic value depends on bilirubin levels: a retrospective multicenter cohort study. (1528-1140 (Electronic)).

28. Yin G, Ma B, Zhou B, Wu J, You L, Xie R. Inflammatory response after different ablation strategies for paroxysmal atrial fibrillation. Heart Surg Forum. 2020;23(5):E703-11.

29. Haran C, Gimpel D, Clark H, McCormack DJ. Preoperative neutrophil and lymphocyte ratio as a predictor of mortality and morbidity after cardiac surgery. Heart Lung Circ. 2020;30(3):414-8.

30. Berkovitch A, Younis A, Grossman Y, Segev S, Kivity S, Sidi Y, Beinart R, Goldenberg I, Maor E. Relation of neutrophil to lymphocyte ratio to risk of incident atrial fibrillation. Am J Cardiol. 2019;123(3):396-401.

31. Liu Y, Lv H, Tan R, An X, Niu XH, Liu YJ, Yang X, Yin X, Xia YL. Platelets promote Ang II (Angiotensin II)-induced atrial fibrillation by releasing TGF- $\beta 1$ (transforming growth factor- $\beta 1$ ) and interacting with fibroblasts. (1524-4563 (Electronic)).

32. Wang $H$, Han J, Wang Z, Yin Z, Liu Z, Jin Y, Han H. A prospective randomized trial of the cut-and-sew Maze procedure in patients undergoing surgery for rheumatic mitral valve disease. J Thorac Cardiovasc Surg. 2018;155(2):608-17.

33. Ad N, Holmes SD, Rongione AJ, Massimiano PS, Fornaresio LM. Does surgical ablation energy source affect long-term success of the concomitant cox maze procedure? Ann Thorac Surg. 2017;104(1):29-35.

34. Nomani H, Saei S, Johnston TP, Sahebkar A, Mohammadpour AH. The efficacy of anti-inflammatory agents in the prevention of atrial fibrillation recurrences. Curr Med Chem. 2021;28(1):137-51.

\section{Publisher's Note}

Springer Nature remains neutral with regard to jurisdictional claims in published maps and institutional affiliations.
Ready to submit your research? Choose BMC and benefit from:
- fast, convenient online submission
- thorough peer review by experienced researchers in your field
- rapid publication on acceptance
- support for research data, including large and complex data types
- gold Open Access which fosters wider collaboration and increased citations
- maximum visibility for your research: over 100M website views per year

At BMC, research is always in progress.

Learn more biomedcentral.com/submissions 